\title{
Ultra-Smooth $\mathrm{Ru}(0001)$ Films as Templates for Ceria Nanoarchitectures - Supplementary Online Material
}

Marc Sauerbrey, ${ }^{\dagger}$ Jan Höcker, $^{\dagger}$ Meikel Wellbrock, ${ }^{\dagger}$ Marco Schowalter $^{\dagger}{ }^{\text {Jon-Olaf }}$ Krisponeit, $^{\dagger}$ Knut Müller-Caspary, ${ }^{\dagger}$ Andreas Rosenauer, ${ }^{\dagger} \ddagger$ Gang Wei, ${ }^{\top}$ Lucio Colombi Ciacchi, ${ }^{\top}, \ddagger$ Jens Falta, ${ }^{\dagger, \ddagger}$ and Jan Ingo Flege ${ }^{*, \dagger}$

$\dagger$ Institute of Solid State Physics, University of Bremen, Otto-Hahn-Allee, 28359 Bremen $\ddagger$ MAPEX Institute for Materials and Processes, University of Bremen, Bremen, Germany IBremen Center for Computational Materials Science, Am Fallturm 1, 28359 Bremen

E-mail: flege@ifp.uni-bremen.de

\section{S1 Growth video}

The LEEM time lapse video S1 shows the growth of cerium oxide on sputter-deposited epitaxial $\mathrm{Ru}$ films at a temperature of about $850^{\circ} \mathrm{C}$. An electron energy of $9.0 \mathrm{eV}$ was chosen to achieve an optimal contrast between the substrate and the ceria islands. In the beginning of the movie one can clearly observe the ceria nucleation process at the Ru surface defects. Then the islands develop their perfectly triangular shape and exhibit a predominant lateral growth. One can also clearly see the strong preference of the azimuthal orientation of the islands. At a minority of the Ru surface defects, dark spots emerge, indicating the concomitant growth of a different ceria phase (presumably ceria(100)). 


\section{S2 Merge video}

The LEEM time lapse video shows the merging of two similar-sized ceria islands at a temperature of about $850^{\circ} \mathrm{C}$ and an electron energy of $9.0 \mathrm{eV}$. As soon as the two islands get in direct contact with each other, the area between the islands is rapidly filled to again form an equilateral triangle. However, the growth is hindered by a defect (marked with red circle). Interestingly, no grain boundary or any other indication of lattice mismatch or height difference can be observed in the contact region of both islands. The only indication that the large island originated from two smaller ones are the two dark spots on the island marking the two initial nucleation centers.

\section{S3 Merge image sequence}

The LEEM image sequence S3 has been recorded at a temperature of about $850^{\circ} \mathrm{C}$ and at an electron energy of $9.0 \mathrm{eV}$. It shows the merging of two ceria islands of significantly different surface area. The same tendency to form a larger equilateral triangle as shown in S2 is observed. However, now the original outline of both islands remains visible, either in form of a step edge or a grain boundary. This could most likely be caused by a difference in the initial island heights. AFM measurements provides confirmation that islands with larger surface area are slightly higher than smaller ones (see main text). 

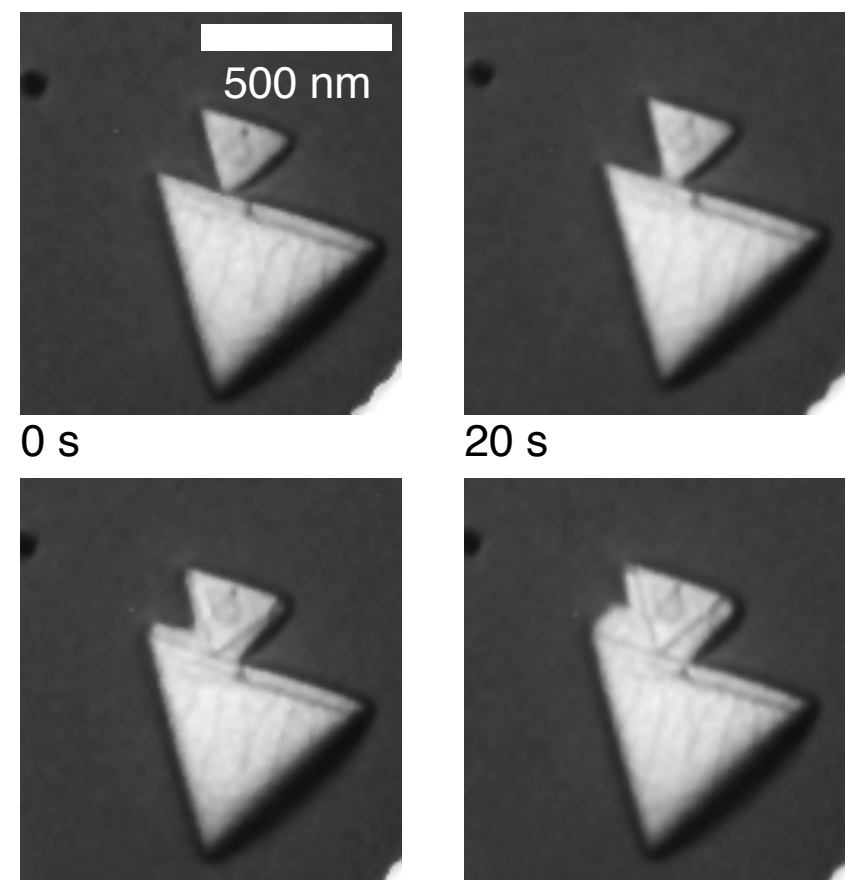

$58 \mathrm{~s}$

$20 \mathrm{~s}$

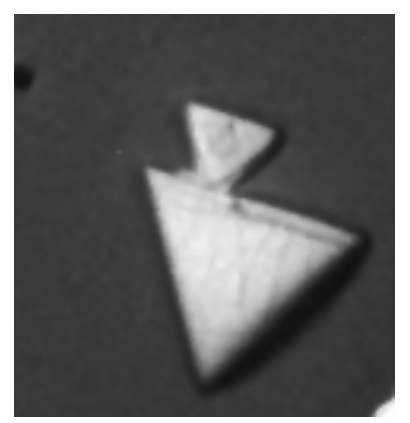

$38 \mathrm{~s}$
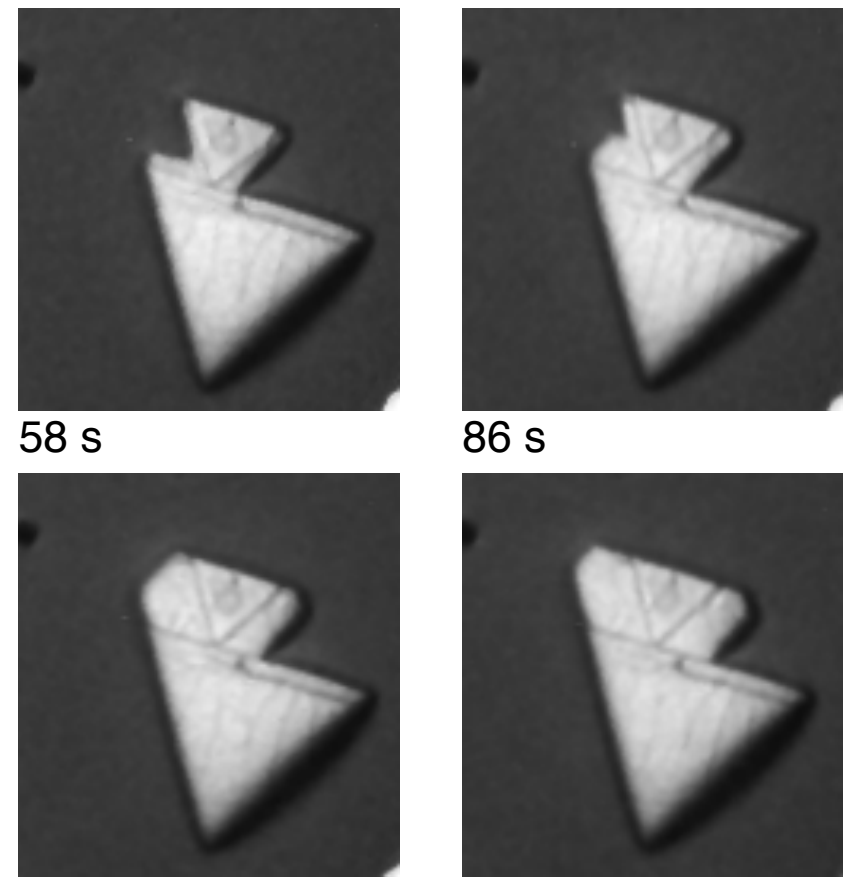

$123 \mathrm{~s}$

$86 s$

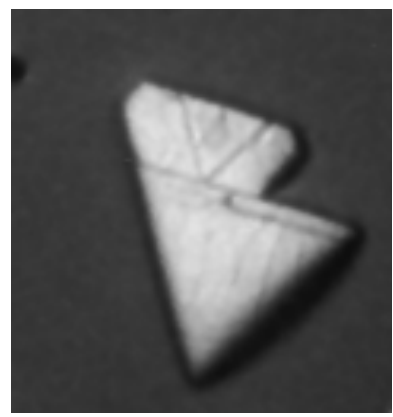

$153 \mathrm{~s}$

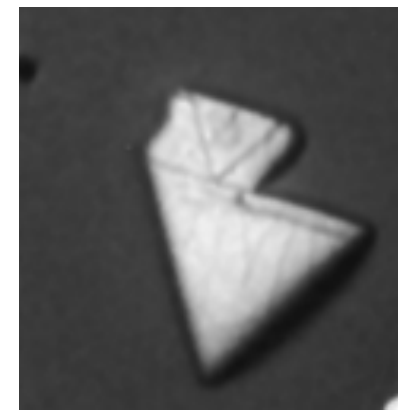

$104 \mathrm{~s}$

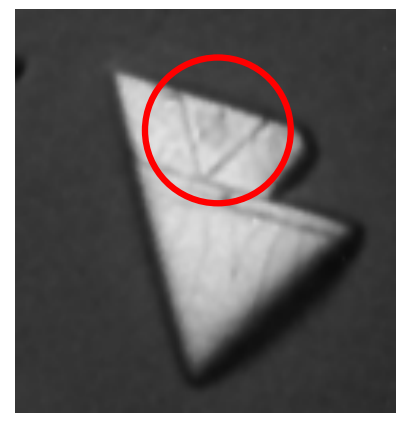

$177 \mathrm{~s}$

Figure S3: LEEM time-lapse sequence showing the merging process of two ceria(111) islands of differing size. 